\title{
The Correlation of Surgical Margins and Outcome Of Soft Tissue Sarcomas of The Extremities and Trunk in Hospital Sultan Ismail, Johor Bahru
}

\author{
Muhamad Firdaus bin Zainudin ${ }^{1}$ \\ ${ }^{1}$ Department of orthopaedics, Traumatology and Rehabilitation, Kulliyyah of \\ Medicine, International Islamic University Malaysia
}

Presenter: Muhamad Firdaus bin Zainudin

Introduction: Patients with soft tissue sarcoma are treated following the standardized guidelines, however it is not guaranteed patient will remain free from local recurrence (LR) and metastasis. This study was conducted to determine the association between microscopic marginal status with local recurrence, metastasis and the survival of the patient. Materials and Methods: This retrospective study was conducted from January 2015 to December 2017 in Hospital Sultan Ismail, Johor Bahru. All patients underwent complete standadized resection of the tumour and had been follow up for 5 years. An operating and follow-up records of 70 cases who fulfill the inclusion and exclusion criteria were collected and analysed using Pearson chi square test and Kaplan-Meier curves. Results: Of the 70 patients, 52 were males and 18 were females. The age of the patients were between 16 and 73 years with the means age of 48.8 years old. Sixty eight patients underwent limb salvage surgery and 2 had amputation. 42 of them had positive post-operative microscopic surgical margin (PMSM) while 28 were negative. Local recurrence was noted in 28 and metastasis in 30 patients. Patient who had PMSM has higher risk to get local recurrence and metastasis compared to patient with negative margin ( $p$-value $<0.001$ ). The overall median survival was 23 months. Overall survival rates for 3 years was $80 \%$ and for 5 years was $65 \%$. Conclusion: In our study, patient with positive post-operative microscopic surgical margin had higher possibility to develop local recurrence, metastases and decrease overall survival rate. 